\title{
Failed ERCP in Extrahepatic Cholangiocarcinoma with Obstructive Jaundice and Cholangitis: An Alternative Approach
}

\author{
Amit Sahu ${ }^{1 *}$ and Arpita Sahu ${ }^{2}$ \\ ${ }^{1}$ MD, Fellowship in Interventional NeuroRadiology, Vascular and Interventional \\ Radiology, India \\ ${ }^{2} \mathrm{MD}$, Fellowship in NeuroImaging, India \\ *Corresponding Author: Amit Sahu, Wockhardt Hospital, Mumbai, India.
}

Received: May 12, 2020

Published: July 01, 2020

(C) All rights are reserved by Amit Sahu and Arpita Sahu.

\begin{abstract}
Cholangiocarcinoma accounting for $2 \%$ of all human malignancies is a rare malignant neoplasm of the biliary tract epithelium. Early stages, being asymptomatic, may go unnoticed and by the time it manifests, the disease process is advanced and usually unresectable. The most common presentation is obstructive jaundice with a lot them progressing to biliary sepsis, septicemia, liver failure and death. Chemotherapy and radiotherapy are ineffective in inoperable tumours. Biliary drainage, either endoscopic or percutaneous, remains the mainstay of palliation in these tumours. Endoscopic cannulation retrogradely across the tumour tissue is difficult and may often prove futile. Percutaneous transhepatic biliary drainage and biliary stenting is a viable, rewarding palliative option with high technical success and low complication rates.
\end{abstract}

Keywords: Percutaneous Transhepatic Biliary Stenting; Percutaneous Transhepatic Biliary Drainage; PTBD; Failed ERCP; Cholangiocarcinoma; Malignant Biliary Obstruction

\section{Introduction}

Cholangiocarcinoma is a rare malignant neoplasm of the biliary tract epithelium, accounting for less than $2 \%$ of all human malignancies [1]. Cholangiocarcinoma is associated with poor patient outcomes. It is difficult to diagnose and is usually fatal, due to its late clinical presentation and the absence of effective non-surgical therapeutic modalities. The incidence of cholangiocarcinoma peaks in patients aged 50 - 70 years with a slight male predisposition [2]. Most patients have unresectable disease at the time of diagnosis and usually die within $6-12$ months from the cancer cachexia, liver failure and biliary sepsis. The 5 -year survival rate is low (about $5 \%$ ) and has remained almost unchanged over last 20 years. Surgical resection is the only curative option and radical resection has improved outcomes, although it is also associated with high perioperative morbidity and mortality rates [3]. Chemotherapy and radiotherapy have been ineffective for patients with inoperable tumours [4].
Biliary stenting for biliary decompression is the mainstay of palliation for non-resectable patients, those with recurrence, and those who refuse surgical treatment [5].

\section{Case Report}

An 82-year-old male presented with jaundice and fever. Contrast enhanced CT scan of the abdomen revealed dilated intrahepatic biliary radicles along with dilated common bile duct in proximal $2 / 3^{\text {rd }}$ beyond which a heterogeneously enhancing intraluminal mass was identified in the common bile duct (Figure 1a-1c).

The patient underwent endoscopic retrograde cholangio-pancreatography (ERCP) under general anaesthesia for biliary drainage by palliative stenting. However, the guidewire cannulation of the common bile duct could not be achieved due to the mass. A repeat ERCP two days later also proved futile (Figure 1d). The patient was subsequently referred for percutaneous transhepatic 
biliary drainage (PTBD). A right sided external-internal PTBD was performed under local anaesthesia. The completely occluding distal CBD mass was traversed antegradely with the guidewire followed by placement of an $8.3 \mathrm{~F}$ ring biliary drainage catheter (Cook Medical) (Figure 1e). A self expanding nitinol metallic stent was eventually placed across the distal CBD mass (Eluminex, Bard) for internal drainage to achieve palliation (Figure 1f). There was good antegrade flow of contrast noted after biliary stenting into the duodenum. The icterus and fever subsequently resolved following percutaneous interventional management.

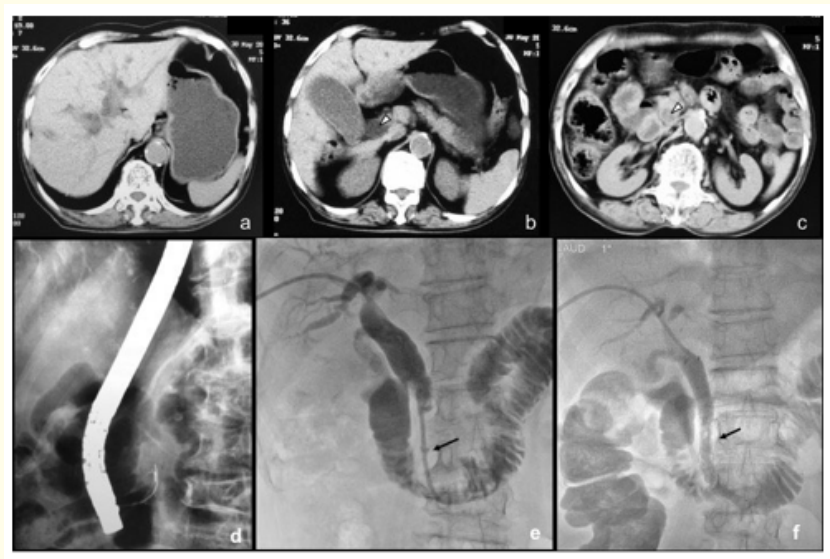

Figure 1: Axial CECT abdomen showing a) Gross right and left intrahepatic biliary dilatation; b) Dilated gall bladder and proximal common bile duct (arrowhead); c) Enhancing intraluminal mass occupying the distal common bile duct (arrowhead); d) ERCP shows contrast opacification of the pancreatic duct but no contrast in the common bile duct and biliary radicles owing to complete obstruction, the cannulation of the bile duct across the mass could not be achieved; e)

Subsequent right sided antegrade PTBD under local anesthesia:

8.3F external-internal pigtail drainage catheter has been positioned across the mass (arrows) into the duodenum. Note the dilated CBD, cystic duct and biliary radicles proximal to the mass;

f) Percutaneous metallic biliary stenting has been performed across the mass with good luminal gain (arrow) and unobstructed antegrade flow into the duodenum. The proximal biliary radicles have decompressed. Catheter was then removed for palliative internal drainage.

\section{Discussion}

Cholangiocarcinoma is a devastating malignancy presenting late in course, is difficult to diagnose, and is associated with a high mortality [4]. It is difficult to diagnose in part because of its relative rarity, and because it is clinically silent until it becomes an advanced disease with obstructive symptoms. The worldwide incidence of cholangiocarcinoma has risen over the past three decades [5].

Its incidence and prevalence are low. It appears from the sixth decade of life and there is slight male predominance [2].

Etiology of cholangiocarcinoma is unknown but conditions like ulcerative colitis, primary sclerosing cholangitis, chronic hepatolithiasis, bile duct adenoma and papillomatosis, congenital hepatic fibrosis and Caroli's disease may lead to the increased incidence. Opisthorchis viverrini, Clonorchis sinensis infestation and chronic typhoid carriers are also at increased risk [2].

The major clinical sign of cholangiocarcinoma is obstructive jaundice, which is persistent and progressive. Biological tumour markers are nonspecific: an increased serum level of carcinoembryonic antigen is relevant when associated with an increased level of CA 19-9 or CA-125 [6].

Biliary obstruction is potentially fatal because of the adverse events associated like reduced immunity, impaired phagocytosis, depressed Kupffer cell function and reduced bile salts arriving at the gut, which may lead to endotoxemia, septicemia and renal failure.

Most of the patients presenting with malignant obstructive jaundice are in advanced stage and almost $70-80 \%$ of such tumours are unresectable at the time of diagnosis [7].

Although surgical resection offers the only hope for cure, most patients are found to have unresectable disease on initial presentation and have an extremely grim prognosis. This has led to an emphasis on the role of palliative care, with relief of biliary obstruction, in the management of these patients. Surgical bypass was once the primary means of palliation of jaundice in these group of patients but in the last 2 decades it has been superseded by less invasive and less morbid nonsurgical procedures such as percutaneous and endoscopic biliary stent placement [8].

Palliation of malignant obstruction not only relieves the patient of itching and jaundice but also reduces the risk of infection and septicemia and brings about an improvement in the quality of life $[7]$. 
Palliative biliary drainage by ERCP for malignant biliary obstruction is unsuccessful in 3 - $10 \%$ cases. Percutaneous transhepatic biliary drainage in such situations is a good alternative [9]. Technical success rate for percutaneous biliary drainage and stenting varies from 86 to $100 \%$ with 30 -day mortality rate $1 \%-49 \%$ and complication rate being $6 \%-56 \%$ [7].

In a meta-analysis by Zhao., et al. they concluded that PTBD may be associated with a better therapeutic success rate for the management of malignant biliary tract occlusion and has lower incidence of cholangitis than endoscopic biliary drainage while the overall complication rate, pancreatitis rate, and 30-day mortality of the two procedures being similar [10]. Patients undergoing metallic stent placement have been reported to live 93 to 420 days longer [7]. PTBD \& Percutaneous metallic biliary stent placement can be safely considered as a first line of bili-ary drainage procedure

\section{Conclusion}

Extrahepatic cholangiocarcinoma usually presents late in the course of disease when it is beyond the purview of curative treatment. It is important to relieve these patients of malignant biliary obstruction to avoid complications that reduce quality of life and may also be life threatening. Endoscopic and percutaneous biliary drainage and stenting are the two palliative options available. Surgery being more invasive and associated with higher morbidity and mortality is generally avoided in such circumstances. In situations when endoscopic drainage it not indicated, fails and/or when the patients cannot be subjected to general anaesthesia, IR treatment in the form of percutaneous biliary drainage and biliary stenting can be performed. It is associated with high technical success and low 30-day mortality and complication rates making it an effective treatment option for palliation of malignant biliary obstruction. Percutaneous biliary drainage should thus be offered as a primary (first line) or secondary means of palliation for management of obstructive jaundice - the decision largely depending on the extent of tumour involvement and associated complications, clinical condition of patient and local availability of expertise.

\section{Bibliography}

1. Parker SL., et al. "Cancer statistics, 1996". CA: A Cancer Journal for Clinicians 46 (1996): 5-27.

2. Khan SA., et al. "Guidelines for the diagnosis and treatment of cholangiocarcinoma: consensus document". Gut 51 (2002): VI1-VI9.
3. Yusoff AR., et al. "Survival analysis of cholangiocarcinoma: a 10 -year experience in Malaysia". World Journal of Gastroenterology 18 (2012): 458-465.

4. Khan SA., et al. "Cholangiocarcinoma". Lancet 366.9493 (2005): 1303-1314.

5. Olnes MJ and Erlich R. "A review and update on cholangiocarcinoma". Oncology 66 (2004): 167-179.

6. Acalovschi M. "Cholangiocarcinoma: risk factors, diagnosis and management". Romanian Journal of Internal Medicine 42.1 (2004): 41-58.

7. Sabharwal T and Adam A. "Biliary tract cancers: extrahepatic management". Geschwind JFH, Dake MD. Abhrams Angiography: Interventional Radiology, third edition. Philadelphia. Lippincott Williams and Wilkins (2013): 163-175.

8. Abu-Hamda EM and Baron TH. "Endoscopic management of cholangiocarcinoma”. Seminars in Liver Disease 24.2 (2004): 165-175.

9. Singh Virendra. "Decompression of Malignant Biliary Obstruction After Failed ERCP: To EUSBD and Not to PTBD?". Digestive Diseases and Sciences 60.2 (2015): 288-289.

10. Zhao XQ., et al. "Comparison of percutaneous transhepatic biliary drainage and endoscopic biliary drainage in the management of malignant biliary tract obstruction: a meta-analysis". Digestive Endoscopy 27.1 (2015): 137-145.

\section{Assets from publication with us}

- Prompt Acknowledgement after receiving the article

- Thorough Double blinded peer review

- Rapid Publication

- Issue of Publication Certificate

- High visibility of your Published work

Website: www.actascientific.com/

Submit Article: www.actascientific.com/submission.php

Email us: editor@actascientific.com

Contact us: +919182824667 